\title{
SELF-CONSISTENT MODELING OF ELECTRODE MOTION IN A MODEL CIRCUIT BREAKER
}

\author{
R. FUCHS \\ Institute for Energy Technology, HSR University of Applied Sciences Rapperswil, 8640 Rapperswil, Switzerland \\ roman.fuchs@hsr.ch
}

\begin{abstract}
Numerical simulations of low-voltage circuit breakers require a coupled solution of gas flow, electromagnetism, electrical circuit, and other aspects. Including electrode motion is challenging because the computational grid is deformed and data is to be exchanged among dedicated solvers. A central issue is to keep them synchronized. This is addressed with a single framework that allows for a continuously morphing grid and accounting for the cumulative effects of mechanics, Lorentz force, and gas pressure. It is shown that gas pressure has negligible effect.
\end{abstract}

Keywords: circuit breaker, electrical arc simulation, self-consistent, rigid body motion.

\section{Introduction}

Low-voltage circuit breakers are designed to carry electrical current in normal operation as well as safely interrupt short-circuit currents in case of failures. In case of a fault, the contacts are opened by means of mechanical actuators and/or electromagnetic forces available due to fault current. This contact opening inevitably leads to an electrical arc. Depending on geometrical and other factors, the arc is ultimately driven into a deion chamber with splitter plates, in which it is elongated and cooled until extinction.

Contact opening can be a major design aspect in the current interruption process and numerical simulations should allow for including this effect appropriately and efficiently. In [1], the computational domain was deformed and remeshed to accommodate for the motion of the opening contact; however, the motion as well as current were not computed but followed input values obtained from experiment. Later, it was shown in $[2,3]$ that numerical simulations should include contact motion appropriately, especially if large contact gaps and contact motion are studied right in front of splitter plates. The adopted workflow consisted of creating a sequence of meshes that correspond to various contact positions. Contact position was calculated by a separate ordinary differential equation. While the meshes remained constant without any deformation, they are swapped frequently to follow contact position. However, the numerical framework involved three codes that must be kept synchronized and being a challenging task. [3] The same approach has been recently adopted in [4].

This contribution presents a self-consistent and seamless implementation of contact motion for a three dimensional model geometry of a low-voltage circuit breaker. The moving contact is assumed to be subject to a mechanical actuator represented as a torsional spring for simplicity, the Lorentz force that becomes important at larger current values, and we account for plasma pressure that acts on the moving contact sur- face. Additionally, we include an electrical circuit that defines electrical boundary conditions for the circuit breaker. In contrast to [1-4], our software framework allows for a continuous deformation and event-based remeshing. Data interpolation and exchange among dedicated solvers is inherently supported in a single user interface, that results in a uniform setup process. An earlier version of the same software framework was used in $[5,6]$ with an advanced plasma model; however, details on specifications of rigid body motion and electrical boundary conditions are not given.

\section{Numerical Simulation Model}

A model circuit breaker (see figure 1) is considered that consists of a fixed electrode, a rotationally opening bridge, and five U-shaped splitter plates enclosed in an insulating box with venting openings. The virtual geometry is designed to be of comparable size to a real device; for reference, the enclosure size is $47 \mathrm{~mm} \times 36 \mathrm{~mm} \times 24 \mathrm{~mm}$. The ambient gas is dry air at atmospheric pressure and gas flow is considered in a box of size $69 \mathrm{~mm} \times 49 \mathrm{~mm} \times 36 \mathrm{~mm}$ with pressure outlets in all directions and solid bodies located centrally. The moving electrode is $2.5 \mathrm{~mm}$ thick and the fixed electrode cross-section is $2 \mathrm{~mm} \times 5 \mathrm{~mm}$. Splitter plates are $1.5 \mathrm{~mm}$ thick and separated in $y$-direction by the same distance. A symmetry plane is applied at $z=0$. The model circuit breaker is thought to be installed serially in an electrical circuit (see figure 2) that consists of a voltage source $\left(V_{\mathrm{rms}}=230 \mathrm{~V}, 50 \mathrm{~Hz}\right)$, a resistor $R=2 \mathrm{~m} \Omega$, and an inductor $L=25 \mu \mathrm{H}$.

We solve the magnetohydrodynamic equations for a thermal plasma in local thermodynamic equilibrium (LTE), i.e., we consider the Navier-Stokes equations

$$
\begin{gathered}
\partial_{t}(\rho)+\nabla \cdot(\rho \mathbf{u})=0, \\
\partial_{t}(\rho \mathbf{u})+\nabla \cdot(\rho \mathbf{u} \otimes \mathbf{u})=-\nabla p+\mathbf{f}_{\mathrm{L}},
\end{gathered}
$$




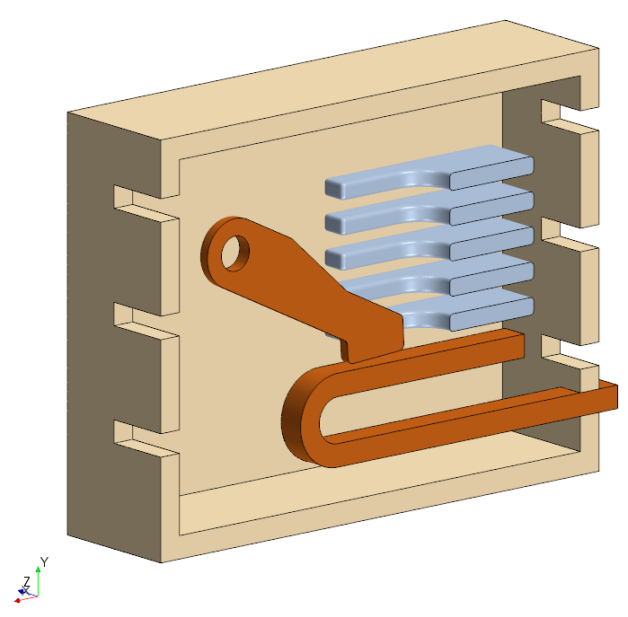

Figure 1. Model geometry of a low-voltage circuit breaker, cut at symmetry plane $z=0$.

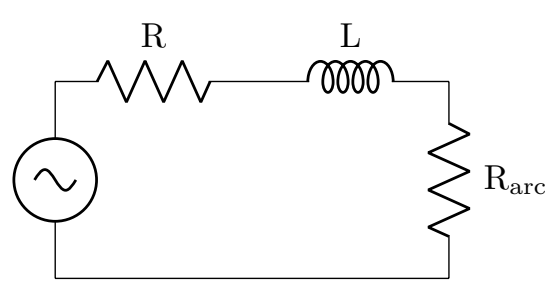

Figure 2. Electrical circuit.

$$
\begin{aligned}
\partial_{t}\left(\rho e_{\mathrm{tot}}\right)+\nabla & \cdot\left(\left(\rho e_{\mathrm{tot}}+p\right) \mathbf{u}\right) \\
& =S_{\mathrm{ohm}}+\nabla \cdot(\lambda \nabla T)-\nabla \cdot \mathbf{q}_{\mathrm{rad}},
\end{aligned}
$$

together with Maxwell's equations in low-frequency limit in $A$ - $\phi$ formulation, i.e.,

$$
\begin{gathered}
\nabla \times \mu^{-1} \nabla \times \mathbf{A}=\mathbf{J}, \\
\nabla \cdot \mathbf{J}=0 .
\end{gathered}
$$

In these equations, $\rho$ stands for gas density, $\mathbf{u}$ gas velocity, $p$ gas pressure, $e_{\text {tot }}$ total energy, $T$ gas temperature, $\mathbf{q}_{\text {rad }}$ radiative heat flux, $\mathbf{J}$ electric current density, B magnetic flux density, $\mu$ magnetic permeability, $\sigma$ electrical conductivity, and $\mathbf{A}$ magnetic vector potential. Moreover, we have by definition $\mathbf{B}=\nabla \times \mathbf{A}$ and $\mathbf{E}=-\nabla \phi$, and Ohm's law $\mathbf{J}=\sigma \mathbf{E}$, where $\mathbf{E}$ denotes electric field and $\phi$ is the electric potential. The gas flow is considered as laminar. The system of equations is coupled via the source terms for Lorentz force density $\mathbf{f}_{\mathrm{L}}=\mathbf{J} \times \mathbf{B}$ and Ohmic heating source density $S_{\mathrm{ohm}}=\mathbf{J} \cdot \mathbf{E}$. Radiative heat flux $\mathbf{q}_{\mathrm{rad}}$ is calculated using the Discrete Ordinate method (see, e.g., [7]) with six frequency bands defined by the ionization energies of nitrogen [8]. Band-averaged absorption coefficients were computed by Planck averaging from spectral data provided by [9]. Thermodynamic and transport properties of dry air plasma are taken from [10]. Material data for electrodes and splitter plates are those of copper and iron, respectively, with relative permeability $\mu_{\mathrm{Fe}, \mathrm{rel}}=1 \times 10^{4}$.
We account for voltage drop in arc roots on the anode and cathode following [8],

$$
U_{a / c}(J)=\frac{a \tilde{J}+b \tilde{J}^{d}}{c+\tilde{J}^{d}}, \quad \tilde{J}=e J
$$

with $a=5 \times 10^{8}, c=9 \times 10^{14}, d=2, e=5$, and $b=$ 5 on the anode and $b=10$ on the cathode. Comparing to [8], we use modified values for coefficients $a, c$, and $e$, so that maximum voltage drop on anode and cathode are reduced to $11.2 \mathrm{~V}$ and $14.7 \mathrm{~V}$, respectively. Hence, the arc is able to run faster into the deion chamber since new arc spots are formed more easily on the splitter plates. The corresponding heat source is included at the plasma-solid interface. This difference in voltage drop modeling is assumed to be of minor importance towards the aim of this study, since we also neglect evaporation and erosion of any solid bodies.

The bridge is assumed to move as a rigid body with rotation axis parallel to $z$-axis. Total torque is computed by the contributions of a torsional spring, gas pressure of the plasma acting on the electrode surface, and Lorentz force density inside the electrode body:

$$
\begin{aligned}
& \boldsymbol{\tau}_{\text {tot }}=\boldsymbol{\tau}_{\mathrm{S}}+\boldsymbol{\tau}_{\mathrm{P}}+\boldsymbol{\tau}_{\mathrm{L}}, \quad \boldsymbol{\tau}_{\mathrm{S}}=-k\left(\alpha-\alpha_{\mathrm{r}}\right) \hat{\mathbf{z}}, \\
& \boldsymbol{\tau}_{\mathrm{P}}=\int_{A} \mathbf{r} \times(-p \mathbf{n}) \mathrm{d} A, \quad \boldsymbol{\tau}_{\mathrm{L}}=\int_{V} \mathbf{r} \times \mathbf{f}_{\mathrm{L}} \mathrm{d} V .
\end{aligned}
$$

The torsional spring constant $k=0.2 \mathrm{~N} \mathrm{~m} \mathrm{rad}^{-1}$ and relaxation angle $\alpha_{\mathrm{r}}=30 \mathrm{deg}$ are defined such that the bridge would open to $\alpha_{\max }=45 \mathrm{deg}$ in $2.5 \mathrm{~ms}$, at which it would be gradually slowed down before hitting the enclosure wall. The natural angular frequency of the torsional spring is $\omega_{\mathrm{n}}=\sqrt{k / I}$ where $I=$ $2.75 \times 10^{-7} \mathrm{~kg} \mathrm{~m}^{2}$ denotes moment of inertia of the moving electrode. If torsional spring was the only acting force, the rotation angle of the moving electrode with respect to $z$-axis is given by

$$
\alpha_{\mathrm{S}}(t)=\alpha_{\mathrm{r}}\left(1-\cos \left(\omega_{\mathrm{n}} t\right)\right)
$$

Initial position of the electrode bridge is defined with a gap of $2 \mathrm{~mm}$ to the fixed electrode. Arc ignition is modeled by a cylinder of radius $1 \mathrm{~mm}$ located centrally between the contacts with electrical conductivity set to $\sigma=1 \times 10^{4} \mathrm{~S} \mathrm{~m}^{-1}$ for $20 \mu \mathrm{s}$. For simplicity, voltage source is assumed to be at peak voltage.

The outlined model is implemented in the numerical framework of Simcenter STAR-CCM+ (v2019.1). The equations for gas flow, electric potential, and radiative heat transfer are solved with finite volume method, and the magnetic vector potential is formulated in finite elements because of the discontinuous magnetic permeability. Therefore, the computational domain is discretized with two meshes, a polyhedral grid for the FV solvers and a tetrahedral grid for the FE solver, with maximum cell sizes $0.5 \mathrm{~mm}$ and $2 \mathrm{~mm}$, respectively. The meshes are refined at solid-fluid interfaces including prism layers. Timestep size is 
$1 \mu \mathrm{s}$. After each timestep, electric current density $\mathbf{J}$ is mapped to the tetrahedral grid, the magnetic vector potential is solved for, and magnetic flux density $\mathbf{B}$ is mapped back to the polyhedral grid. The domain can be remeshed whenever a specific criterion is met. Here, it is triggered if the upper electrode has moved $0.5 \mathrm{deg}$ since the last remeshing event, or if the simulation had to be restarted. The rigid body dynamics and electrical circuit are also implemented in the user interface.

\section{Results}

Results are presented for time until $t=400 \mu \mathrm{s}$. Figure 3 shows arc voltage and current, as well as current through the lowest two splitter plates. We see that arc current rises almost linearly as defined by the electrical circuit. After ignition, arc voltage is at $26 \mathrm{~V}$ comparing well to the sum of voltage drop on anode and cathode, and increases gradually until $t=200 \mu \mathrm{s}$. This is explained by plasma cloud expanding from the ignition region into a volume bounded by the moving electrode tip, the fixed electrode and the lower two splitter plates. We note that a fraction of the current flows through the first splitter plate, and after $t=200 \mu \mathrm{s}$, we see that electrical current starts to flow through the second splitter plate. At the same time value, we also note that arc voltage increases more substantially. This is related to the plasma gas expanding upwards and arc root formation at second splitter plate. Since the focus of this work is on electrode motion modeling, we limit the discussion of plasma flow to this level as it becomes more complex.

Figure 4 shows total torque on the moving electrode and its contributing terms due to torsional spring, Lorentz force, and plasma pressure. We see that torsional spring and Lorentz force are dominant, while plasma pressure yields a small contribution at arc ignition and quickly becomes negligible. Torsional spring yields an almost constant torque because of mass inertia and therefore relatively slow angular motion in the time interval considered. As current increases, Lorentz force becomes gradually more important and its torque exceeds that of torsional spring after $t=300 \mu \mathrm{s}$.

To support the understanding of the data discussed above, the spatial distribution of plasma pressure and Lorentz force density at $t=100 \mu$ s are shown in figures 5 and 6 , respectively. We see that the pressure wave due to arc ignition has already expanded from electrode tip towards splitter plates. Hence, torque due to plasma pressure is small. However, Lorentz force density increases gradually as total current increases and the self-induced magnetic field due to electric flux. Hence, their contribution to total torque becomes more important.

Figure 7 shows rotation angles due to total torque as obtained by the simulation and that of the analytical model in equation (4). We clearly see that torque due to Lorentz force results in faster electrode motion.

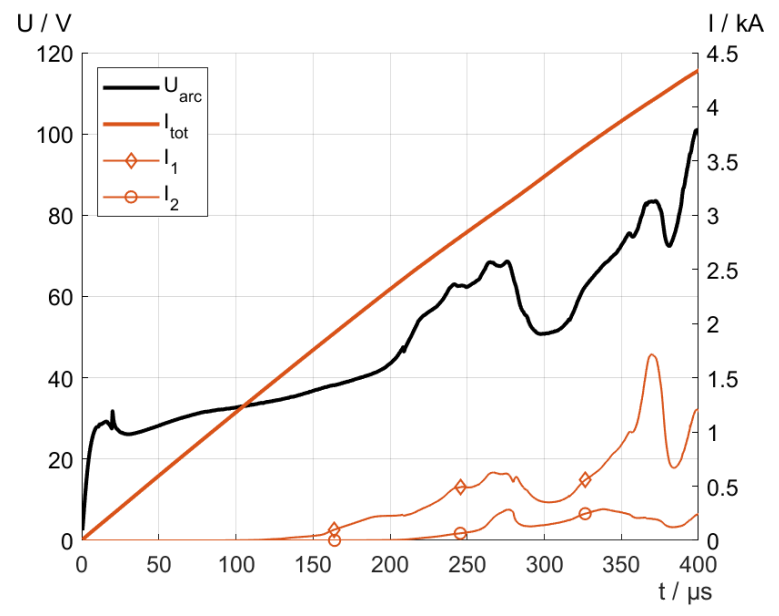

Figure 3. Arc voltage and current in circuit breaker. The lines $I_{1}$ and $I_{2}$ show electrical current through the lowest splitter plates.

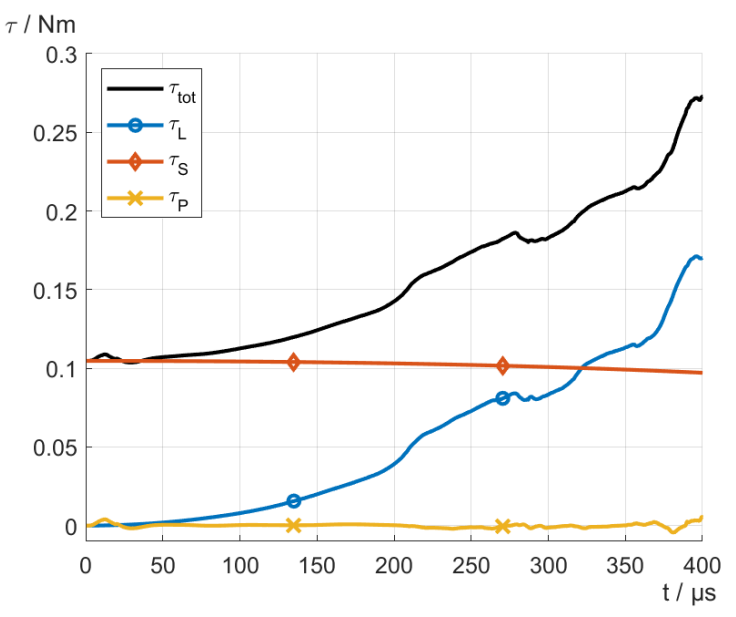

Figure 4. Total torque on the moving electrode, with contributions from Lorentz force density, torsional spring, and plasma pressure.

This effect becomes more pronounced with increasing time values.

\section{Discussion and Conclusions}

This contribution presents a self-consistent model for electrode motion in a virtual low-voltage circuit breaker. The results show that torque is mainly due to torsional spring and Lorentz force. Plasma pressure has negligible influence on rigid body dynamics because of its small torque value and its short interaction time. This does not mean that accurate plasma modeling is of minor importance; it is of uttermost importance because plasma gas flow and electromagnetic fields are directly affected. The mechanical actuator should be modeled appropriately because it affects rigid body dynamics and its interactions with arc plasma. Therefore, a self-consistent arc model should be used that allows to compute electrical current and voltage defined by the circuit elements, as well as to compute rigid body motion including the effects of mechanics and Lorentz force. 


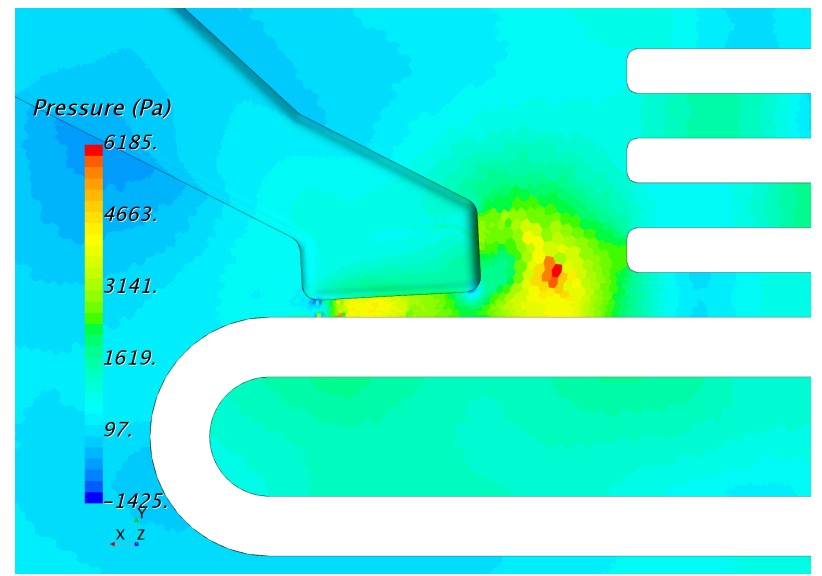

Figure 5. Plasma pressure at $t=100 \mu \mathrm{s}$.

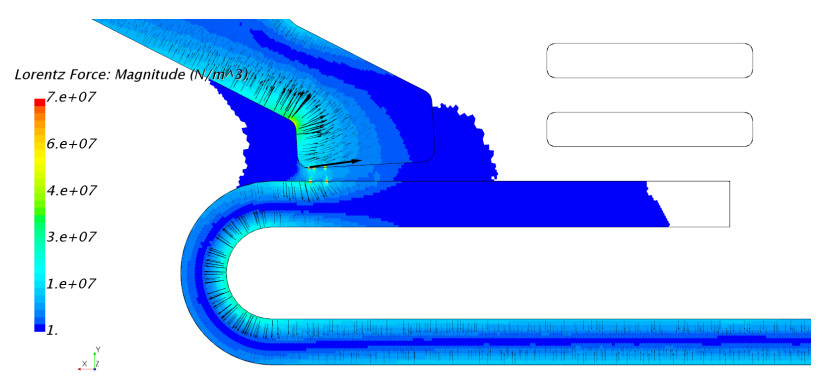

Figure 6. Lorentz force density at $t=100 \mathrm{\mu s}$.

In contrast to previous work, the presented model is implemented with a continuously deforming mesh that is remeshed whenever needed. The user interface allows to define rigid body motions directly inside the framework without particular programming. It also inherently allows for data exchange among the solvers. As a consequence, the workflow for preparing simulations with complex body motions and interactions of the plasma with electrical circuit may be eased.

Future work will extend this study to longer simulation time, additional plasma physics including copper vapor due to electrode erosion and wall ablation, nonlinearity of $B-H$ curve of iron and further modeling techniques to ease user interaction with the software. Experiments would serve ideally to complement this study.

\section{Acknowledgements}

This work was supported by Siemens PLM Software. The author thanks Dr Boris Klauderic and Dr Angelo Limone for many valuable discussions.

\section{References}

[1] Y. Wu, M. Rong, Z. Sun, X. Wang, F. Yang, and X. Li. Numerical analysis of arc plasma behaviour during contact opening process in low-voltage switching device. Journal of Physics D: Applied Physics, 40(3):795-802, 2007. doi : 10.1088/0022-3727/40/3/016.

[2] C. Rümpler, A. Zacharias, and H. Stammberger. Lowvoltage circuit breaker arc simulation including contact

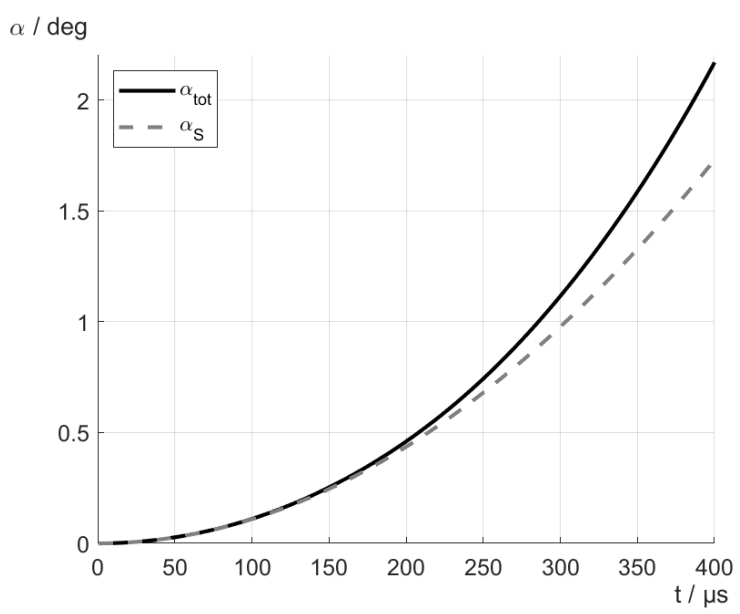

Figure 7. Rotation angle due to total torque and compared to that due to torsional spring only.

arm motion. In F. Berger, editor, The 27th International Conference on Electrical Contacts, pages 290-294, 2014.

[3] C. Rümpler and V. Naranyanan. Arc Modeling Challenges. Plasma Physics and Technology, 2(3):261-270, 2015.

[4] D. Shin, J. W. McBride, and I. O. Golosnoy. Arc Modeling to Predict Arc Extinction in Low-Voltage Switching Devices. In 2018 IEEE Holm Conference on Electrical Contacts, pages 222-228. IEEE, 14.10.2018 18.10.2018. doi:10.1109/HOLM. 2018.8611712.

[5] Y. Enami and M. Sakata. Simulation of arc in molded-case circuit breaker with metal vapor and moving electrode. In 2013 2nd International Conference on Electric Power Equipment - Switching Technology (ICEPE-ST), pages 1-4. IEEE, 20.10.2013 - 23.10.2013. doi:10.1109/ICEPE-ST . 2013.6804390.

[6] M. Sakata and Y. Enami. Arc Simulation Technology. Fuji Electric Review, 60(3):195-200, 2014.

[7] M. F. Modest. Radiative heat transfer. Academic Press, New York, 3. edition, 2013.

[8] C. Rümpler. Lichtbogensimulation für Niederspannungsschaltgeräte. Dissertation, TU Ilmenau, Ilmenau, 2009.

[9] P. Kloc, V. Aubrecht, M. Bartlova, and O. Coufal. Radiation transfer in air and air-Cu plasmas for two temperature profiles. Journal of Physics D: Applied Physics, 48(5):055208, 2015. doi:10.1088/0022-3727/48/5/055208.

[10] R. N. Gupta, K.-P. Lee, R. A. Thompson, and J. M. Yos. Calculations and curve fits of thermodynamic and transport properties for equilibrium air to $30000 \mathrm{~K}$. N92-11285, NASA, 1991. 